\title{
Uso de lenguaje estandarizado en prácticas formativas de enfermería. Pereira 2011-2015
}

\author{
Use of standardized language on nursing apprenticeships. Pereira 2011-2015 \\ Uso da linguaje padronizada em práticas formativas de enfermagem (Pereira 2011-2015) \\ María Nelcy Muñoz-Astudillo* \\ Yogel Alberto Rúas-Amaya** \\ Mónica Margarita Barón-Castro ${ }^{* * *}$
}

\section{Resumen}

Objetivo: Caracterizar el uso del lenguaje estandarizado en el registro del Proceso de Enfermería a la población atendida durante las prácticas formativas en una Institución Universitaria de Pereira Colombia, durante los años 2011 a 2015. Materiales y Métodos: Estudio descriptivo retrospectivo autorizado por el comité de Ética Institucional. Se ordenaron según frecuencia 30.020 diagnósticos enfermeros, registrados durante los años 2011 a 2015 en el sistema de información de prácticas formativas de enfermería. Se seleccionaron los diagnósticos más frecuentes, sus resultados esperados e intervenciones hasta completar el tamaño de muestra con un $\mathrm{NC}=95 \%$ y error de precisión $=1 \%$. La muestra correspondió a 7276 registros que incluyeron 15 diagnósticos presentes en 4731 personas atendidas. Variables: Características de la población atendida, tipo de práctica formativa y Proceso de Atención de Enfermería (PAE) con taxonomía NANDA-I, NOC y NIC. Resultados: Predomina la atención a la población femenina $(57,4 \%)$, principalmente adultos $(72 \%)$, régimen subsidiado (68\%). Las prácticas se realizan principalmente en áreas clínicas (84\%) de instituciones hospitalarias estatales $(68 \%)$. Se priorizaron diagnósticos 'reales' $(69,3 \%)$ relacionados con los dominios: 'Seguridad/Protección' (22,8\%), 'Eliminación/Intercambio' (21,0\%) y 'Actividad/ Reposo' (21,0\%). NOC más frecuentes en dominios 'Salud fisiológica (63,0\%) y 'Salud funcional '(16,8\%). NIC relevantes en campos de 'Seguridad '(27,4\%), 'Fisiológico complejo '(26,8\%) y 'Fisiológico básico '(24,5\%). Conclusiones: Coherencia en la aplicación de las fases del PAE. Los diagnósticos más frecuentes correspondieron a problemas de salud clínicos, fisiológicos y funcionales. Se requiere fortalecer la formulación y registro de diagnósticos de 'Percepción/cognición' y resultados del dominio 'Psicosocial' que orienten intervenciones educativas en familias y comunidades.

Palabras Clave: Educación en Enfermería, procesos de Enfermería, NANDA, NIC, NOC, práctica, Formación profesional.

\section{Autor de correspondencia}

* Enfermera. Magister en Enfermería en Salud Pública. Magister en Enfermería Materno Infantil. Docente Facultad de Salud. Fundación Universitaria del Área Andina. E-mail: mmunoz@areandina.edu.co. Orcid: (D) http://orcid.org/0000-0002-9310-3310. Pereira. Colombia.

** Ingeniero de Sistemas. Magister en Informática Educativa. Doctor en Educación. Docente Facultad de Ingeniería y Ciencias Básicas. Fundación Universitaria del Área Andina. E-mail: yruas@areandina.edu.co Orcid: (D) http://orcid.org/0000-0003-30042474 Pereira. Colombia

*** Enfermera. Magister en Enfermería con énfasis en Adulto y Anciano. Docente Facultad de Salud. Fundación Universitaria del Área Andina. E-mail: mbaron@areandina. edu.co ORCID (D) http://orcid.org/00000001-6310-7364 Pereira. Colombia.

Este es un artículo bajo la licencia CC BY (https://creativecommons.org/ licenses/by/4.0/) लc () 
ISSN-PRINT

1794-9831

E-ISSN 2322-7028

Vol. 16 No. 1

Ene - Abr 2019

Cúcuta, Colombia

\begin{abstract}
Objective: Characterize the use of standardized language in the nursing records of an attended population during internships in a University of Pereira, Colombia. Materials and Methods: Retrospective descriptive study authorized by the Institutional Ethics Committee. According to frequency, 30,020 nursing diagnoses registered in the information system of nursing internships from 2011 to 2015 were organized. The most frequent diagnoses, their expected results and interventions were selected until completing a sample size of $\mathrm{NC}=95 \%$ and an error precision $=1 \%$. The sample corresponded to 7276 records that included 15 diagnoses present in 4731 of the people attended. Variables: characteristics of the attended population, type of internship and Nursing Process with NANDA-I, NOC and NIC taxonomy. Results: Care predominates on female population (57.4\%), mainly adults $(72 \%)$, of subsidized regime $(68 \%)$. The practices were mainly performed on clinical areas $(84 \%)$ of State healthcare institutions (68\%). 'Real' diagnoses were prioritized (69.3\%) relating to the domains: 'Security/Protection' (22.8\%), 'Elimination/Exchange' (21.0\%), 'Activity/Rest' (21.0\%). The most frequent NOC domains 'Physiological health' (63.0\%) and 'functional health' (16.8\%). Relevant NIC domains on areas of 'security' (27.4\%), 'complex physiological' $(26.8 \%)$ and 'basic physiological' (24.5\%). Conclusions: Coherence in the application of the Nursing Process phases. The most frequent diagnoses corresponded to clinical physiological and functional health problems. It is required to strengthen the formulation and diagnoses records of 'Perception/Cognition' and the results of the 'Psychosocial' domain to orientate educative interventions on families and communities.
\end{abstract}

Keywords: Nursing Education, nursing processes NANDA, NIC, NOC, practice, vocational education.

\begin{abstract}
Resumo
Objetivo: Caracterizar o uso da linguagem padronizada no registro do Processo de Enfermagem à população atendida durante as práticas formativas numa Instituição Universitária de Pereira na Colômbia, durante os anos 2011 a 2015. Materiais e Métodos: Estudo descritivo retrospectivo autorizado pelo comité de Ética Institucional. Ordenaram-se segundo frequência 30.020 diagnósticos de enfermagem, registrados durante os anos 2011 a 2015 no sistema de informação de práticas formativas de enfermagem. Selecionaram-se os diagnósticos mais frequentes, seus resultados esperados e intervenções até completar o tamanho de amostra com um $\mathrm{NC}=95 \%$ e erro de precisão $=1 \%$. A amostra correspondeu a 7.276 registros que incluíram 15 diagnósticos presentes em 4.731 pessoas atendidas. Variáveis: Características da população atendida, tipo de prática formativa e Processo de Atendimento de Enfermagem (PAE) com taxonomia NANDA-I, NOC e NIC. Resultados: Predomina o atendimento à população feminina $(57,4 \%)$, principalmente adultos $(72 \%)$, regímen subsidiado $(68 \%)$. As práticas se realizam principalmente em áreas clínicas $(84 \%)$ de instituições hospitalares estaduais (68\%). Priorizaram-se diagnósticos 'reais' $(69,3 \%)$ relacionados com os domínios: "Seguridade/Proteção" (22,8\%), "Eliminação/Trocas" ' (21,0\%) e “Atividade/ Repouso" (21,0\%). NOC mais frequentes em domínios "Saúde fisiológica" (63,0\%) e "Saúde funcional" (16,8\%). NIC relevantes em campos de "Segurança" (27,4\%), "Fisiológico complexo" (26,8\%) e "Fisiológico básico" $(24,5 \%)$. Conclusões: Coerência na aplicação das fases do PAE. Os diagnósticos mais frequentes corresponderam a problemas de saúde clínicos, fisiológicos e funcionais. Reque-se fortalecer a formulação e registro de diagnósticos de "Percepção/cognição" e resultados do domínio "Psicossocial" que orientem intervenções educativas em famílias e comunidades.
\end{abstract}

Palavras-chave: Educação em Enfermagem, processos de Enfermagem, NANDA, NIC, NOC, prática, Formação professional.

\section{Introducción}

El desarrollo de la disciplina de Enfermería durante mucho tiempo no tuvo un método único para aplicar los conocimientos que se iban generando en su evo- lución, aunque las actividades lo tenían implícito, no era considerado como proceso (1); se piensa que la aplicación más temprana de elementos del Proceso de Enfermería la realizó Florence Nightingale a mediados del siglo XIX (2). En la década de 1950, fue Lidia 
Hall quien describió por primera vez el quehacer de la enfermería como un proceso, pero su valioso aporte no tuvo eco en el ejercicio de la enfermería (3). En 1967, Yura y Walsh publicaron la descripción del proceso de Enfermería en cuatro fases: valoración, planeación, ejecución y evaluación. En 1974 y 1975 Blody y Roy añadieron la fase de diagnóstico (3). Actualmente, el Proceso de Atención de Enfermería (PAE) involucra cinco fases: valoración, diagnóstico, planeación, ejecución y evaluación del cuidado del enfermo.

El PAE se considera a nivel internacional como la base del ejercicio profesional; es un método sistemático, dinámico y humanístico que sirve para consolidar la teoría y la práctica en el cuidado profesional de las experiencias de salud de individuos, familias y comunidades. A partir de la valoración continua de estos sujetos de cuidado, se plantean nuevas situaciones que llevan a enfermeras y enfermeros a realizar las demás fases del proceso para lograr una evolución acorde con los resultados esperados. Hace cuatro décadas los registros de las cinco fases en el mundo eran manuales, gracias al interés de Asociaciones Internacionales de Enfermería en el desarrollo de taxonomías, los avances en sistematización y estandarización del lenguaje para diagnósticos, resultados esperados e intervenciones han facilitado la comprensión de las fases del PAE y sus interacciones, expandiendo su aplicación a diversas áreas de desempeño.

La North American Nursing Diagnosis Association International (NANDA) define el diagnóstico enfermero como un "juicio clínico en relación con una respuesta humana no deseada de una persona, familia, grupo o comunidad, a una afección de salud/proceso vital" (4); los primeros registros se remontan al año 1973 durante la Primera Conferencia Nacional de Clasificación de Diagnósticos de Enfermería en San Luis, Missouri, EEUU y actualmente, el listado de diagnósticos versión 2018-2020 registra 244 diagnósticos vigentes, distribuidos en los 13 dominios de respuestas humanas (4).

Los Resultados esperados y observados en el paciente, sensibles a la práctica enfermera aparecen definidos en la Nursing Outcomes Classification (NOC) como "un estado, conducta o percepción de una persona, familia o comunidad, medido a lo largo de un continuo, en respuesta a una intervención o intervenciones enfermera/s" (5). La utilización de los resultados esperados se inició en la década de 1960 con Aydelotte con el propósito de valorar la calidad de los cuidados enfermeros; posteriormente se han desarrollo numerosos esquemas de ajustes y medidas. En la actualidad, la clasificación NOC es la taxonomía más difundida; en la quinta edición del año 2013 se reconocieron 500 resultados vigentes, agrupados en 7 dominios y 32 clases (5).

De igual manera, las intervenciones fueron definidas por la Nursing Interventions Classification (NIC) como "todo tratamiento basado en el conocimiento y juicio clínico que realiza un profesional de enfermería para favorecer el resultado esperado del paciente" (6). El equipo de la NIC se fundó en 1987 y en su sexta edición del año 2013 aparecen 554 intervenciones enfermeras vigentes, agrupadas en 7 campos y 30 clases para facilitar su uso (6).

Se debe precisar que, la taxonomía ha sido ampliamente acogida en varios países. Desde el año 1996, uno de sus principales promotores es la Asociación Española de Nomenclatura, Taxonomía y Diagnósticos de Enfermería (AENTDE) (7). En el año 2001 se creó la " $N N N$ Alliance" una relación virtual de colaboración entre la NANDA-I y la Universidad de Iowa con el objetivo de "avanzar en el desarrollo, la verificación y el perfeccionamiento del lenguaje estandarizado" (4), trabajando en diagnósticos y resultados esperados e intervenciones.

También, la estandarización ha contado con la participación y compromiso de profesionales de Enfermería en el mundo entero, tanto para la generación como para la validación de diagnósticos y resultados esperados y cada día incursiona con mayor fuerza en los currículos de formación profesional. En Brasil, en el año 2013 se compartieron experiencias en enseñanza, asesoramiento, gestión e investigación en los escenarios de la práctica profesional (8). Por otra parte en la "Escola de Enfermagem da Universidade Federal do Rio Grande do Sul" el grupo de enfermería viene realizando un arduo trabajo de desarrollo taxonómico, sistematización y divulgación del PAE del suroccidente del país, liderando la capacitación en otras Instituciones (9). Actualmente, el Proceso de Enfermería ha evolucionado hacia su tercera generación, caracterizada por la eficacia del cuidado, medida por los resultados esperados (10).

Los términos NANDA-I, NIC y NOC (NNN) son reconocidos por la American Nurses Association (ANA). Están incluidos en el Metathesaurus de la 
ISSN-PRINT

1794-9831

E-ISSN 2322-7028

Vol. 16 No. 1

Ene - Abr 2019

Cúcuta, Colombia
Biblioteca Nacional de Medicina para Lenguaje Médico Unificado y en el Índice Acumulativo de la Literatura de Enfermería (CINAHL). También están mapeados en SNOMED (Nomenclatura Sistemática de Medicina) y registrados en la Organización de informática médica Health Level Seven Internacional (HL7) (11).

En Colombia, la Universidad Industrial de Santander fue pionera en el uso de la taxonomía, e inició los procesos de sistematización de prácticas formativas de enfermería en el año 2001(12). El uso de la taxonomía NANDA-I, NOC, NIC en Programas de Enfermería lo promueve la Asociación Colombiana de Facultades de Enfermería (ACOFAEN); adquirió relevancia desde el año 2012 por su utilización en la evaluación de competencias profesionales en las pruebas estatales Saber PRO (13). De igual forma, se han realizado Congresos Internacionales sobre Lenguaje estandarizado en los años 2013, 2015 (11) y 2017 (14).

En la institución donde se realizó este estudio, desde el año 2011, docentes y estudiantes registran el PAE con la taxonomía NANDA-I, NOC, NIC en el Sistema de Información de Prácticas Formativas de Enfermería; este sistema fue creado y validado por docentes de la misma institución y registrado ante el Ministerio del Interior de Colombia (15). En este trabajo se describen datos registrados para la población atendida durante los años 2011-2015.

Conocer el uso de la clasificación NNN durante las prácticas formativas es importante y pertinente para el Programa de Enfermería. Los hallazgos permitirán analizar la efectividad del aprendizaje estudiantil, conocer la correspondencia entre teoría y práctica para retroalimentar el proceso curricular e identificar necesidades de tutoría, capacitación, desarrollo y evaluación en PAE. Se logrará una línea de base para gestionar la Relación Docencia-Servicio (16), mostrando indicadores de práctica basada en la evidencia.

\section{Objetivos}

\section{Objetivo General}

Caracterizar el uso del lenguaje estandarizado en el registro del Proceso de Enfermería a la población atendida durante las prácticas formativas en una Institución Universitaria de Pereira Colombia, durante los años 2011 a 2015.

\section{Objetivos Específicos}

- Caracterizar la población atendida según género, ciclo vital, régimen de aseguramiento en salud, procedencia y estrato de la vivienda.

- Distribuir la población atendida según tipo de institución y práctica formativa.

- Identificar los Diagnósticos - NANDA-I registrados con mayor frecuencia, los Resultados esperados-NOC e intervenciones-NIC para estos diagnósticos, en una muestra de tamaño representativo de la población atendida.

\section{Materiales y Métodos}

Es un estudio descriptivo, transversal, de tipo exploratorio, realizado en una Institución Universitaria de Pereira-Colombia. Se analizó la información registrada en el Software del Sistema de Información de Prácticas Formativas de Enfermería F_PAE.

Población y muestra: entre los años 2011 y 2015 se encontraron registrados en el software mencionado, 30.020 diagnósticos enfermeros, correspondientes a 15.738 personas atendidas durante las prácticas formativas de Enfermería. El muestreo fue intencional. Los registros se ordenaron de mayor a menor frecuencia de diagnóstico. De este listado se seleccionaron los de mayor frecuencia hasta completar un tamaño de muestra representativo de la población, con un $\mathrm{NC}=95 \%$ y un error de precisión del $1 \%$, el cual correspondió a 7.276 registros que incluyeron los 15 diagnósticos más frecuentes presentes en 4.731 personas atendidas.

Variables: la población atendida, se caracterizó según género, ciclo vital, régimen de aseguramiento en salud y procedencia; el tipo de práctica formativa se distribuyó según Institución y asignatura práctica. El Proceso de Atención de Enfermería se clasificó con taxonomía NANDA-I, NOC, NIC. Los 7.276 registros de los 15 diagnósticos-NANDA más frecuentes se clasificaron según tipo, dominio y clase. Para estos 15 diagnósticos se encontraron 5.879 registros de resultados esperados, los cuales estaban distribuidos en 45 resultados-NOC que se clasificaron según dominio y clase.

Para los 15 diagnósticos más frecuentes se habían registrado 9.185 intervenciones-NIC, las cuales fueron clasificadas según campo de intervención. 
Análisis: se utilizó SPSS V.20 y se establecieron proporciones para cada variable.

Estudio autorizado por el Representante Legal de la Institución Universitaria. Los registros fueron codificados y tratados de manera anónima. Se obtuvo la aprobación del Comité de Ética Institucional, bajo el Código CV2017-P23

\section{Resultados}

\section{Características de la Población atendida}

Los 15 diagnósticos enfermeros más frecuentes estuvieron presentes en 7.276 registros, correspondientes a 4.731 personas atendidas que se caracterizan en la Tabla 1.

Tabla 1. Características sociodemográficas de la población atendida

\begin{tabular}{lcc}
\hline Género & Frecuencia & Porcentaje \\
\hline Femenino & 2876 & 60,8 \\
Masculino & 1855 & 39,2 \\
Ciclo vital & Frecuencia & Porcentaje \\
\hline Lactantes & 237 & 5,0 \\
Preescolares & 416 & 8,8 \\
Escolares & 222 & 4,7 \\
Adolescentes & 449 & 9,5 \\
\hline Adulto joven & 1145 & 24,2 \\
Adulto maduro & 1112 & 23,5 \\
\hline Adulto mayor & 1150 & 24,3 \\
\hline Régimen de aseguramiento & Frecuencia & Porcentaje \\
\hline Subsidiado & 3217 & 68,0 \\
\hline Contributivo & 1197 & 25,3 \\
\hline Vinculado & 317 & 6,7 \\
\hline Procedencia & Frecuencia & Porcentaje \\
\hline Área urbana. Vivienda estrato medio & 1873 & 39,6 \\
\hline Área urbana. Vivienda estrato bajo & 1992 & 42,1 \\
\hline Área rural & 866 & 18,3 \\
\hline
\end{tabular}

Fuente: Sistema de Información de prácticas formativas de Enfermería F PAE

En la población atendida durante las prácticas formativas se observó un predominio de población femenina $(60,8 \%)$, de adultos $(72 \%)$, de régimen subsidiado $(68 \%)$ y de área urbana $(81,7 \%)$.

\section{Distribución de la población atendida según la} práctica formativa
El 84,1\% de la población incluida en los 15 diagnósticos más frecuentes fue atendido en escenarios de práctica clínica y el 15,9\% en área comunitaria. En la Tabla 2 se aprecia la distribución según Institución en convenio de Relación Docencia-Servicio (RDS) y tipo de práctica formativa del Plan de estudios del Programa. 
ISSN-PRINT

1794-9831

E-ISSN 2322-7028

Vol. 16 No. 1

Ene - Abr 2019

Cúcuta, Colombia

Tabla 2. Distribución de la muestra poblacional según Práctica formativa

\begin{tabular}{lcc}
\hline Institución en convenio de RDS & Frecuencia & Porcentaje \\
\hline Hospitales y Clínicas de alta complejidad & 1859 & 39,3 \\
\hline Hospitales de mediana complejidad & 932 & 19,7 \\
Hospitales de baja complejidad & 875 & 18,5 \\
Comunidades Barriales & 615 & 13,0 \\
Centros especializados y grupos terapéuticos & 449 & 9,5 \\
Muestra poblacional & 4731 & $100 \%$ \\
Tipo de práctica formativa & Frecuencia & Porcentaje \\
\hline Cuidado de Enfermería al adulto y la familia II & 929 & 19,6 \\
Cuidado de Enfermería al adulto y la familia I & 823 & 17,4 \\
Principios científicos del cuidado de Enfermería & 736 & 15,6 \\
Cuidado de Enfermería al niño y la familia & 674 & 14,2 \\
Cuidado de Enfermería a la familia & 596 & 12,6 \\
Cuidado de Enfermería a la mujer, madre y RN & 513 & 10,8 \\
Promoción de la salud y la familia & 194 & 4,1 \\
\hline Cuidado de Enfermería al adolescente y la familia & 161 & 3,4 \\
Gerencia de servicios de Salud & 105 & 2,2 \\
\hline Muestra poblacional & 4731 & 100,0 \\
\hline
\end{tabular}

Fuente: Sistema de Información de prácticas formativas de Enfermería F_PAE

La mayoría de las personas incluidas en la muestra había sido atendida en Instituciones de alta complejidad $(39,3 \%)$ y los registros correspondían principalmente a las prácticas de cuidado de enfermería al adulto $(37 \%)$. La coherencia entre el área clínica de atención, la institución de alta complejidad y el Cuidado de Enfermería al adulto y familia II permiten deducir que las áreas clínicas especializadas en cuidado del adulto son muy importantes para las prácticas formativas de profesionales de enfermería.

\section{El proceso de Atención de Enfermería.}

\section{Diagnósticos de Enfermería- Taxonomía NANDA}

- Tipo de diagnóstico: de los 15 diagnósticos de enfermería más frecuentes durante las prácticas formativas, el 69,4\% corresponde a diagnósticos reales, el 15,6\% a diagnósticos de riesgo y el
15,1\% a diagnósticos de bienestar (Tabla 3).

- Distribución por dominios: 'Seguridad/protección' (22,8\%), 'Eliminación/ Intercambio' (21\%), Actividad/Reposo (21\%), 'Confort' (13,2\%), 'Rol/Relaciones' (7,1\%), 'Promoción de la salud' (6,6\%) ', Nutrición' (5,9\%), 'Sexualidad' (1,3\%) y 'Afrontamiento/ Tolerancia al estrés' $(1,1 \%)$. No fueron priorizados diagnósticos en los dominios de 'Percepción/cognición', 'Autopercepción', 'Principios vitales' ni 'Crecimiento y desarrollo'.

- Distribución de diagnósticos según Clase. Predominio de 'Infección', seguido de 'Función Respiratoria', 'Actividad/ Ejercicio', 'Confort Físico', 'Relación Familiar', 'Mantenimiento de la salud', 'Ingestión', 'Reproducción' y 'Afrontamiento'. 


\section{.}

Tabla 3. Diagnósticos Enfermeros registrados con mayor frecuencia durante las prácticas formativas

\begin{tabular}{|c|c|c|c|}
\hline Diagnósticos Enfermeros & Frecuencia & $\% *$ & Tipo de Diagnóstico \\
\hline Riesgo de infección & 1067 & 14,7 & Riesgo \\
\hline Deterioro del intercambio de gases & 969 & 13,3 & \multirow{5}{*}{ Real } \\
\hline Dolor agudo & 962 & 13,2 & \\
\hline Deterioro de la movilidad física & 805 & 11,1 & \\
\hline Deterioro de la integridad cutánea & 589 & 8,1 & \\
\hline Deterioro de la eliminación urinaria & 560 & 7,7 & \\
\hline Disposición para mejorar los procesos familiares & 516 & 7,1 & \multirow{2}{*}{ Bienestar } \\
\hline Disposición para mejorar la gestión de la propia salud & 483 & 6,6 & \\
\hline Patrón respiratorio ineficaz & 450 & 6,2 & \multirow{3}{*}{ Real } \\
\hline Desequilibrio nutricional: ingesta inferior a las necesidades & 363 & 5,0 & \\
\hline Disminución del gasto cardiaco & 201 & 2,8 & \\
\hline Disposición para mejorar el proceso de maternidad & 97 & 1,3 & Bienestar \\
\hline Ansiedad & 77 & 1,1 & \multirow{2}{*}{ Real } \\
\hline Perfusión tisular periférica ineficaz & 72 & 1,0 & \\
\hline Riesgo de nivel de glucemia inestable. & 65 & 0,9 & Riesgo \\
\hline 15 Diagnósticos enfermeros más frecuentes & 7276 & 100,0 & \\
\hline
\end{tabular}

Fuente: Sistema de Información de prácticas formativas de Enfermería F_PAE

Resultados Esperados- Taxonomía NOC

En los 15 diagnósticos más frecuentes se encontraron 5.879 registros de resultados esperados que estaban distribuidos en 45 resultados-NOC. La Tabla 4 pre-

senta la distribución de los registros de esultados esperados según Clase y Dominio.

Tabla 4. Resultados esperados- NOC según Clase y Dominio, para los 15 diagnósticos enfermeros más frecuentes en escenarios de prácticas formativas

\begin{tabular}{|c|c|c|c|}
\hline Clase del Resultado-NOC & Frecuencia & $\% *$ & Dominio \\
\hline Cardiopulmonar & 1341 & 22,8 & \multirow{3}{*}{ Salud Fisiológica } \\
\hline Eliminación & 934 & 15,9 & \\
\hline Integridad Tisular & 760 & 12,9 & \\
\hline Movilidad & 667 & 11,3 & Salud Funcional \\
\hline Conducta de salud & 505 & 8,6 & $\begin{array}{l}\text { Conocimiento y } \\
\text { Conducta }\end{array}$ \\
\hline Bienestar Familiar & 507 & 8,6 & Salud Familiar \\
\hline Neurocognitiva & 319 & 5,4 & Salud Fisiológica \\
\hline Mantenimiento de la Energía & 262 & 4,5 & Salud Funcional \\
\hline Control del riesgo y seguridad & 237 & 4,0 & $\begin{array}{l}\text { Conocimiento y } \\
\text { Conducta }\end{array}$ \\
\hline Regulación metabólica & 184 & 3,1 & \multirow{3}{*}{ Salud Fisiológica } \\
\hline Digestión y Nutrición & 114 & 1,9 & \\
\hline Respuesta inmune & 49 & 0,8 & \\
\hline Total, registros NOC para los 15 diagnósticos más frecuentes & 5879 & 100,0 & \\
\hline
\end{tabular}

Fuente: Sistema de Información de prácticas formativas de Enfermería F_PAE

El dominio registrado con mayor frecuencia fue

'Salud Fisiológica' (63\%), seguido de 'Salud Funcional' (15,8\%), 'Conocimiento y conducta en salud' (12,6\%) y 'Salud Familiar' (8,6\%). La distribución de los 5.879 registros de NOC según Clases y Dominios de clase muestra que las Cla- 
ISSN-PRINT

$1794-9831$

E-ISSN 2322-7028

Vol. 16 No. 1

Ene - Abr 2019

Cúcuta, Colombia ses 'Cardiopulmonar', 'Eliminación', 'Integridad tisular' fueron las más frecuentes. No se encontraron registros de NOC correspondientes al dominio de 'Salud Psicosocial'.

Intervenciones de Enfermería- Taxonomía NIC
Para los 15 diagnósticos más frecuentes se registraron 9.185 intervenciones de enfermería. Los NIC registrados con mayor frecu encia fueron: Protección contra las infecciones, monitorización de signos vitales, cambios de posición, manejo del dolor y oxigenoterapia (Tabla 5).

Tabla 5. Intervenciones de Enfermería para los 15 Diagnósticos Enfermeros registrados con mayor frecuencia

\begin{tabular}{lccc}
\hline Intervenciones -NIC más frecuentes & Frecuencia & $\% *$ & Campos de intervención \\
\hline 6550 Protección contra las infecciones & 1458 & 15,9 & Seguridad \\
\hline 6680 Monitorización de signos vitales & 1059 & 11,5 & \\
0840 Cambio de posición & 931 & 10,1 & Fisiológico básico \\
1400 Manejo del dolor & 901 & 9,8 & \\
3320 Oxigenoterapia & 828 & 9,0 & \\
3590 Vigilancia de la piel & 825 & 9,0 & Fisiológico complejo \\
2300 Administración de Medicación & 813 & 8,9 & \\
7140 Apoyo a la familia & 741 & 8,1 & Familia \\
\hline 5270 Apoyo emocional & 723 & 7,9 & Conductual \\
1050 Alimentación & 238 & 2,6 & Fisiológico básico \\
1100 Manejo de la Nutrición & 180 & 2,0 & \\
8274 Fomentar el desarrollo: niño & 170 & 1,9 & Familia \\
7150 Terapia familiar & 161 & 1,8 & \\
\hline 8880 Protección de riesgos ambientales & 157 & 1,7 & Comunidad \\
\hline Registros NIC para los 15 Diagnósticos más frecuentes & 9185 & 100,0 & \\
\hline
\end{tabular}

Fuente: Sistema de Información de prácticas formativas de Enfermería F_PAE

La distribución de Intervenciones mostró mayor proporción así: Seguridad (27,4\%) 'Fisiológico complejo' (26,8\%), 'Fisiológico básico' (24,5\%), 'Familia' (11,7\%), 'Conductual' (7,9\%) y 'Comunidad' (1,7\%). Son escasos los registros en el campo Conductual y de Comunidad; no aparecen priorizados registros de intervenciones educativas, ni de apoyo al sistema sanitario.

\section{Discusión}

La sistematización del PAE con lenguaje estandarizado NANDA-NOC-NIC se considera una experiencia de apropiación tecnológica muy importante y de gran utilidad en la formación de docentes y estudiantes para las instituciones universitarias; posibilita a los estudiantes superar algunos obstáculos en la comprensión del método, descritos en otros estudios (1719) y avanzar en el desarrollo científico de la Enfermería.

Del mismo modo, las prácticas formativas de enfermería se realizan con mayor frecuencia en hospitales y clínicas; esto refleja, al igual que lo expresado por Caligiore (20), que en la formación de los profesionales de Enfermería sobresale el enfoque curativo frente al preventivo.

La mayoría de las prácticas se realizan en Instituciones de Salud del Estado, a donde acuden principalmente pacientes del régimen subsidiado. Esto explica en parte la caracterización de la población atendida y las instituciones participantes. Aunque las mayores proporciones de adultos en este estudio se justifican, porque de las nueve asignaturas prácticas que tiene el Plan de estudios, seis se realizan con población de adultos (21), este grupo poblacional fue predominante en varios estudios sobre PAE en prácticas formativas (22-27), lo que lleva a pensar en sujetos de cuidado y escenarios de práctica selectivos que pueden posibilitar el logro de la mayoría de las competencias para el desempeño profesional.

Algo similar ocurre con lo encontrado por GonzalesVilla y otros (22), quienes validan la Guía de prácticas 
clínicas como herramienta educativa; en este estudio las proporciones de diagnósticos de riesgo y de bienestar son bajas y no se identifican diagnósticos de los dominios de percepción, cognición y autopercepción. Adicionalmente, a la escasez de NOC en el dominio de Salud Psicosocial y de NIC en el campo conductual y de comunidad, se muestra de manera reiterativa el predominio del componente clínico y de la visión biologicista del cuidado, frente a lo psicosocial, emocional y cultural, en la formación de profesionales de Enfermería.

También, se identifican varios diagnósticos reales que son relevantes en otros contextos de práctica formativa: riesgo de infección (22-26), dolor agudo (22-26), deterioro de la integridad tisular (22-26), deterioro de la movilidad física (22-26), ansiedad (22-26), desequilibrio nutricional por defecto $(22,24,26)$ y patrón respiratorio ineficaz $(25,26)$. De igual manera, los Resultados- NOC (24) y las Intervenciones- NIC (24-26) prevalentes, son hallazgos compartidos. De este análisis surge una inquietud: ¿Es posible estandarizar los planes de estudio de Enfermería en áreas de conocimiento específicas de formación profesional?

Los registros del Proceso de atención de Enfermería durante las prácticas formativas reflejan la exposición del estudiante al desafío de tomar decisiones diagnósticas y terapéuticas coherentes con las situaciones vividas por los pacientes, que estén acordes con los tiempos de interacción y los recursos disponibles, según los escenarios de práctica. Resultados que son diferentes a lo encontrado por Jara (18), quien refirió disparidades en el desarrollo del método por parte de los estudiantes; es por eso, que en este trabajo se observó consistencia entre los diagnósticos, los resultados esperados y las intervenciones de enfermería registradas con mayor frecuencia, durante el período en estudio.

Se debe precisar, que los diagnósticos durante las prácticas formativas se concentran principalmente en la población de adultos con procesos crónicos que requieren atención especializada, en condiciones clínicamente estables. Estos diagnósticos posiblemente, son registrados con mayor frecuencia porque afectan en forma directa el bienestar físico, exigen atención inmediata y posibilitan medir tanto los resultados obtenidos con el cuidado enfermero durante la jornada de práctica, como las competencias de formación profesional. Datos que son diferente a lo registrado en los trabajos sobre prácticas formativas; es por eso que en la literatura se destaca la validación de diag- nósticos enfermeros realizados por profesionales y con poblaciones específicas, por ejemplo niños (27), pacientes críticos (28)' o pacientes con enfermedades crónicas (29), los cuales se orientan a desarrollar planes de cuidado estandarizado.

De acuerdo con lo planteado por Martínez (19), una de las mayores necesidades que emergen en el escenario de prácticas formativas es la evaluación de las competencias de los estudiantes en PAE integrando teoría y práctica; en este sentido se debe considerar que la perspectiva de quien recibe el cuidado es fundamental para evaluar la integralidad y la pertinencia del proceso educativo. Razón por la cual, la eficacia del cuidado, entendida como la capacidad de lograr el resultado esperado, medida con las escalas propuestas por la taxonomía NOC, debe convertirse en un indicador importante para apoyar la evaluación de las competencias de los estudiantes en los escenarios de práctica (30).

En el mismo sentido de lo expresado, Umaña (31) reafirma la necesidad de formar profesionales de enfermería con competencias en la práctica basada en la evidencia (PBE). García e Ibáñez (32) sistematizaron una experiencia de práctica formativa que da cuenta de la posibilidad de involucrar a los estudiantes en la sistematización de sus propias experiencias de cuidado y en investigaciones que tengan como insumo principal los registros sistematizados del proceso de enfermería.

Una de las mayores limitaciones de este estudio fue el subregistro de los diagnósticos, resultados esperados e intervenciones correspondientes al desempeño en el área comunitaria; así como las dificultades técnicas para el transporte de implementos y equipos hacia algunos escenarios de práctica, lo que explica en parte la escasez de estos registros.

\section{Conclusiones}

- La población atendida durante las prácticas formativas de enfermería, se caracteriza por ser adulta, de género femenino, procedentes de área urbana, pertenecientes al régimen subsidiado de salud, con problemas de salud crónicos agudizados. Los hallazgos permiten deducir que las áreas clínicas especializadas en cuidado del adulto son muy importantes para las prácticas formativas de profesionales de enfermería y que en la formación hay predominio del enfoque curativo frente al preventivo. 
ISSN-PRINT

1794-9831

E-ISSN 2322-7028

Vol. 16 No. 1

Ene - Abr 2019

Cúcuta, Colombia
- El uso del lenguaje estandarizado para el registro del Proceso de enfermería durante las prácticas formativas permite apreciar sus beneficios para el proceso académico porque: mejora la comprensión del método para los estudiantes, permite el seguimiento y retroalimentación de los procesos, se orienta hacia la generación de estándares de desempeño por práctica y ofrece información actualizada para el planteamiento de políticas institucionales en la relación docencia-servicio.

- Los diagnósticos enfermeros reales y los resultados esperados relacionados con el componente biológico/clínico son más frecuentes que los relacionados con el componente social/ comunitario. Esto se explica en parte por la predominancia de los registros de prácticas realizadas en el área clínica. Por tanto, es necesario fortalecer la identificación y el registro de diagnósticos de los dominios de percepción/ cognición y autopercepción, plantear resultados esperados del dominio psicosocial y diseñar intervenciones de tipo educativo en el campo conductual, así como de apoyo al sistema sanitario de la comunidad.

- En el presente estudio se encontró coherencia entre los diagnósticos enfermeros, los resultados esperados y las intervenciones; éstos hallazgos fueron coherentes con la valoración de los usuarios

- Es necesario continuar los análisis de los datos y aprovechar la información disponible para la realización de investigaciones que incluyan la
Práctica basada en la Evidencia y orienten hacia la construcción de instrumentos de evaluación de las prácticas formativas, integrando las competencias del estudiante y la eficacia del cuidado, tomando como referencia principal los indicadores de los resultados esperados.

- Uno de los grandes beneficios del uso del lenguaje estandarizado durante la formación profesional es el mejoramiento de las competencias de las y los futuros profesionales para interactuar con pares del mundo; tal como lo expresan Stephens y Hennefer (33), quienes manifiestan que en procesos de internacionalización, la utilización del mismo lenguaje en la generación de conocimiento, permite evidenciar la diversidad cultural y comparar de manera sistemática el trabajo que se realiza con individuos, familias y comunidades.

\section{Agradecimientos}

A la Fundación Universitaria del Área Andina Seccional Pereira-Colombia por su apoyo logístico y financiero; así como a las instituciones en convenio de relación Docencia- Servicio que posibilitan estos estudios. A la Doctora Patricia Duran por su apoyo durante el proceso de sistematización de las prácticas formativas.

\section{Conflicto de Intereses}

Los autores declaran no tener ningún conflicto de intereses.

\section{Referencias Bibliográficas}

1. Reina NC. El Proceso de Enfermería: Instrumento para el cuidado. Umbral Científico. 2010; (17):1823

2. Rakuom CP, Wagoro MCA, Mirereh JO, Sudi S. Returning Nursing to Nightingale: The Bigger Picture of Mainstreaming Kenya-Nursing Process. J Comm Pub Health Nursing. 2016; 2(4):1-20.

3. Universidad de Antioquia. Historia del Proceso de Atención de Enfermería. Plataforma académica para pregrado y posgrado [Internet] Medellín, 2016. [Consultado 20 marzo 2018] Disponible en http:// aprendeenlinea.udea.edu.co/lms/moodle/mod/page/view.php?id=61913

4. Heather Herdman T, Kamitsuru S. Editores. NANDA International. Diagnósticos Enfermeros: Definiciones y clasificación 2018-2020. 11 edición. España: Thieme; 2017.

5. Moorhead S, Jhonson M, Maas M, Swanson E, editores. Clasificación de resultados de enfermería. (NOC). Medición de Resultados en Salud. 5a ed. España: Elsevier; 2014.

6. Bulechek G, Butcher H, Dochterman J, Wagner C. Clasificación de intervenciones de enfermería (NIC). $6^{\mathrm{a}}$ ed. España: Elsevier; 2014: XVII 
7. Asociación Española de Nomenclatura, Taxonomía y Diagnósticos de Enfermería. AENTDE. (Editores). Jornadas de actualización. 2016

8. Moura-Santos C, David-Lopes MG. Sistematización de la Atención de Enfermería y Responsabilidad social en los escenarios de práctica profesional. [Editorial] Rev Bras Enferm. 2013; 66(5): 647.

9. Almeida MA, Lucena AF, Franzen E, Laurent MC. Processo de enfermagem na prática clínica: estudos clínicos realizados no Hospital de Clínicas de Porto Alegre. Porto Alegre: Artmed; 2011.

10. González-Castillo MG, Monroy-Rojas A. Proceso enfermero de tercera generación. Enferm Univ. 2016; 13(2):124-129.

11. NANDA International. Defining the Knowledge of Nursing. NNN. [Internet] Available in http://www. nanda.org/nanda-i-nic-noc.html

12. Memorias II Congreso Latinoamericano NANDA Internacional. Evolución del Cuidado de Enfermería desde el Lenguaje Estandarizado NANDA-NIC-NOC. Bucaramanga: Universidad Industrial de Santander. 2015. Junio 11-13

13. República de Colombia. Ministerio de Educación. Instituto Colombiano para el fomento de la Educación Superior (ICFES). Estructura general del examen Saber Pro. [Internet] Bogotá; 2016 [consultado 20 de marzo de 2018] Disponible en: http://www.icfes.gov.co/item/1982-modulos-saber-pro-2016-2

14. Universidad Industrial de Santander. Escuela de Enfermería. III Congreso de Proceso de Atención de Enfermería y Lenguaje estandarizado. 22 de septiembre de 2017 [Internet]; [consultado 03-12-2017] Disponible en http://www.congresointernacionalprocesoenfermeriauis2017.com/

15. Barón Castro M, Rúas-Amaya Y, Muñoz-Astudillo MN. Software Sistema de Información Prácticas de Enfermería F_PAE. Fundación Universitaria del Área Andina seccional Pereira Colombia. Registro Ministerio del Interior 13-61-245 obtenido 30 de junio de 2017.

16. Colombia. Decreto 2376 del $1^{\circ}$ de Julio de 2010. Por medio del cual se regula la relación docencia servicio para los programas de formación de talento humano del área de la salud

17. Diaz Aguilar R, Amador-Morán R, Alonso-Uría RM, Campo-González A, Mederos-Dueñas M, OriaSaavedra M. Evaluación del conocimiento del Proceso de Atención de Enfermería: Estudiantes de licenciatura de Enfermería. Facultad «Lidia Doce». 2013 Enferm Univ. 2015; 12(1):36-40

18. Jara Sanabria S, Lizano-Pérez A. Aplicación del Proceso de Atención de Enfermería por estudiantes, un estudio desde la experiencia vivida. Enferm Univ. 2016;13(4):208-215

19. Martínez-Royert, JC. Prácticas evaluativas del proceso de atención de enfermería: una visión de docentes y estudiantes. Rev Cuid. 2017; 8(1):1459-75.

20. Caligiore-Corrales I, Díaz-Sosa JA. Competencias en la atención primaria en salud de los egresados de la carrera de enfermería. Universidad de los Andes. Rev. cienc. cuidad. 2015; 10(2):40-49.

21. Fundación Universitaria del Área Andina Seccional Pereira. Programa de Enfermería. Proyecto Pedagógico del Programa. Plan de estudios 2013. 210 p.

22. González-Villa P, Blanco-Rodríguez JM, Diez-Gómez J, López-Romero MA. Guía de prácticas clínicas: herramienta educativa para la enseñanza de la metodología enfermera. Enfermería Docente [Internet] 2015 [citado 2018 Abr 12];2(104). Disponible en: http://www.revistaenfermeriadocente.es/index. $\mathrm{php} / \mathrm{ENDO} / \mathrm{article} / \mathrm{view} / 25$

23. Alonso K, Silvera S. Aplicación del proceso enfermero en la práctica hospitalaria por los estudiantes de la licenciatura de enfermería de la FETS. Enfermería: Cuidados Humanizados [Revista en Internet] 27 de enero de 2016 [citado 2018 Abr 12]; 2(1). Disponible en: https://revistas.ucu.edu.uy/index.php/ enfermeriacuidadoshumanizados/article/view/780

24. Laguado Jaimes E. Villamizar-Osorio ML. Desarrollo de la estrategia pedagógica "Proyectos Formativos" para fomentar la integralidad del conocimiento disciplinar. Revista Cubana de Enfermería [revista en Internet]. 2016 [citado 2018 Abr 12];32(4) [aprox. 0 p.]. Disponible en: http://www.reven- 
ISSN-PRINT

$1794-9831$

E-ISSN 2322-7028

Vol. 16 No. 1

Ene - Abr 2019

Cúcuta, Colombia fermeria.sld.cu/index.php/enf/article/view/980

25. Escobar-Montoya JI, Muñoz-Astudillo MN. Diseño e implementación del sistema de información para la relación docencia-servicio del Programa de Enfermería en la Universidad Libre. Rev. Cultura del Cuidado. 2009 Dic; 6(2):14-29

26. Ibáñez Alfonso LE, Amaya-Gamarra IC, Céspedes-Cuevas V. Visibilidad de la Escuela de Enfermería en los campos de práctica. Rev. Aquichán. 2005. 29 de agosto; 5(1):8-19

27. Nóbrega RV, Lima da Nóbrega MM, De Lima K. Diagnósticos, Resultados e Intervenções de Enfermagem para crianças na Clínica Pediátrica de um Hospital escola. Rev Bras de Emferm, Brasilia. 2011; 64(3):501-510.

28. Lucena AF, Rivero de G MG, Echer IC., Leite de B AL. Intervenciones de Enfermería utilizadas en la práctica clínica de una Unidad de terapia Intensiva. Rev. Latino-am Enfermagem 2010; 18(5):873-880.

29. Villareo L. Diagnósticos de enfermería en pacientes dados de alta, con enfermedades crónicas, según taxonomía NANDA. Enferm. glob. 2011; (23): 25-29.

30. Gómez Díaz MP, Laguado Jaimes E. Propuesta de evaluación para las prácticas formativas en enfermería. Rev Cuid, 2013; 4(1).

31. Ureña Molina MP, López Medina IM, Pancorbo-Hidalgo PL. Práctica basada en evidencia en estudiantes de enfermería colombianos. Rev. cienc. cuidad. 2017; 14(2):51-64.

32. García Rueda A, Ibáñez Alfonso LE. Sistematización de una experiencia de cuidado con los y las adolescentes. Rev. cienc. cuidad. 2013; 10(2):65-75

33. Stephens M, Hennefer D. Internationalising the nursing curriculum using a Community of InquiryFramework and blended learning. Nurse Education in Practice, 2013; 13(3):170-175. 